\title{
THE SEVEN-INCH TRANSIT CIRCLE and its NEW ZEALAND PROGRAM
}

J. A. Hughes, M. D. Robinson, F. S. Gauss and R. C. Stone

U. S. Naval Observatory

\section{THE SEVEN-INCH TRANSIT CIRCLE}

\section{General Features}

The modernization of the seven-inch transit circle is an important aspect of the envisioned Naval Observatory observing effort. This task has been carried out on the basis of the following desiderata:

1) The basic sensor should be photoelectric

2) Point sources observable at night down to at least $m=10$

3) Venus, Mercury and stars down to $m=4$ observable in the daytime

4) Mars, Jupiter, Saturn, Uranus, Neptune and the Moon observable

5) The Sun must be observable

6) The instrumental parameters: azimuth, level, collimation, nadir, and so on must be determined so as to allow absolute observations

7) The system should operate essentially autonomously, but an observer should be kept in the observing "loop".

It appears that all of these objectives have been achieved by the system now to be briefly described.

\section{The Image Dissector Micrometer (IDM)}

The central feature of the new system consists of an image dissector scanning appropriate raster patterns so as to determine the position of the image of the observed object with respect to reference wires mounted in the prime focus. The double vertical wires and a collimating lens are mounted onto a ball slide driven by a digital velocity servo. The double horizontal wires are mounted directly on the base of the IDM and do not move. Integral bosses are machined into the base and serve as hard zero stops for two Heidenhain 0.1 micron encoders which measure the position of the ball slide. The ground ways against which the slide balls are preloaded are also mounted onto machined surfaces on the base. The intersection of the wires forms a box 32 " square on the sky. The

$$
483
$$

H. K. Eichhorn and R. J. Leacock (eds.). Astrometric Techniques, 483-496.

(C) 1986 by the IAU. 
A. Image Dissector

B. Reimaging Lens

C. Filter Wheel

1. Mirror

2. Interference Filter

3. Polarizer

4. Beam Splitter

5. Clear Aperture
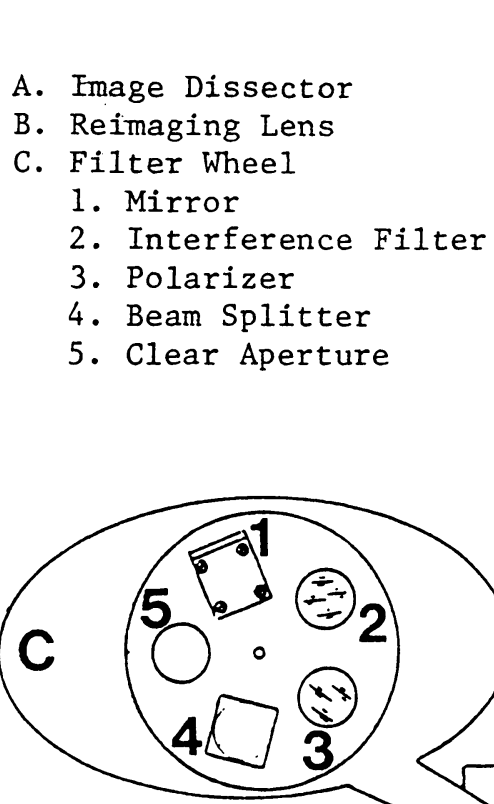

D. Servo Drive

E. Collimating Lens

F. Linear Encoder

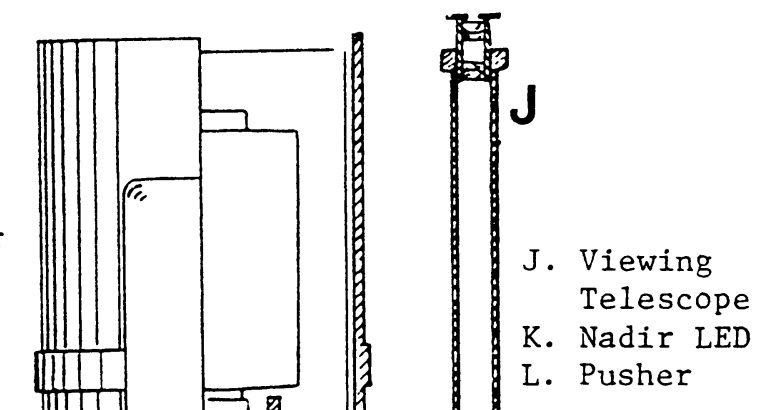

G. Focal Plane Wires

1. Declination Wires

2. Moveable Right Ascension Wires

H. LED Ring

I. Shutter

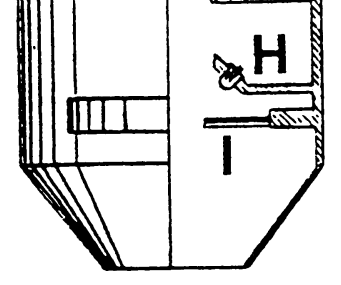

L. Pusher 


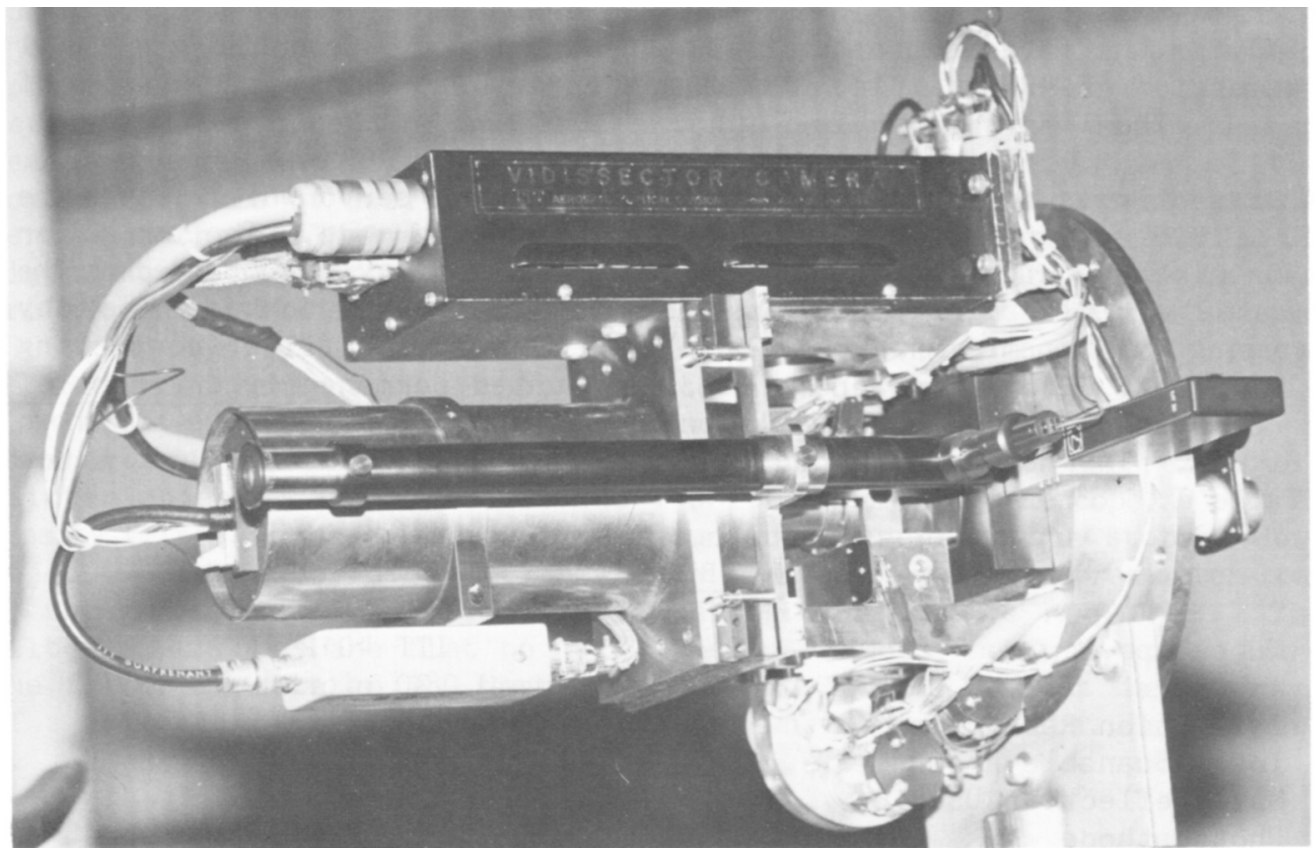

Figure 2 - Image Dissector Micrometer

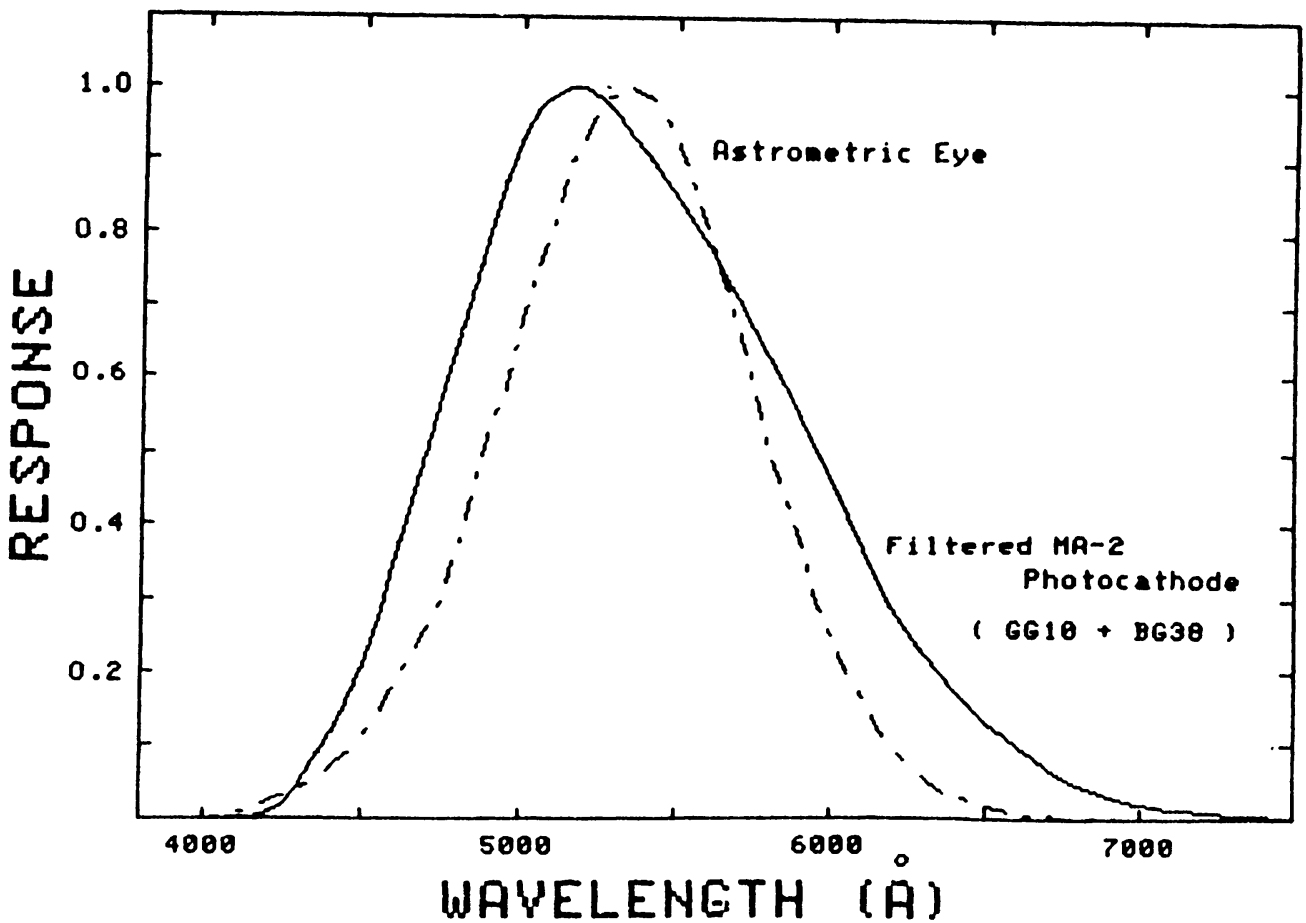

Figure 3 - System Color Response 
center of this box, determined by dissector scans of the re-imaged wires $(2.4 X)$, is the reference datum on the photocathode. Essential structural parts are of 17-4PH stainless steel, secondary supporting parts are of titanium. The IDM is shown in Figures 1 and 2.

The dissector is driven in hour angle by the same servo which drives the ball slide and operates in the photon counting mode. The amplifier /discriminator, high voltage and deflection circuitry are mounted on the IDM. All air/glass interface surfaces are multi-layer coated, thereby increasing the transmission from $25 \%$ to $29 \%$. A sandwich filter consisting of $1 \mathrm{~mm}$ Schott GG10 + 2mm Schott BG38 is mounted permanently in front of the reimaging lens. The system's passband and that of the eye are shown in Fig. 3. The most significant parameters of the dissector are given in Table $I$.

\section{TABLE I}

Tube Type

Aperture

Deflection Resolution

Total Scanable Area

Max. Deflection Used

Photocathode

Max. Quantum Efficiency

Deflection Linearity

Weight Including Magnetic Shielding

$\begin{array}{cr} & \text { ITT } 4012 \mathrm{RP} \\ \text { nominal } & 30 \text { micron sq. } \\ \text { nominal } & 1.5 \mathrm{micron} \\ 5 \times 5 \mathrm{~mm} \\ \tilde{M} 1 \mathrm{~mm} \\ \text { MA2 } \\ >10 \% \\ \sim 0.1 \% \\ 2.3 \mathrm{~kg}\end{array}$

\section{The Telescope Setting Mechanism}

Setting of the telescope in zenith distance is accomplished using coarse and fine rotating mechanisms, and a photoelectric device which counts divisions of the divided circle as they pass a reference point during rotation of the telescope. A ramped stepping motor is the prime mover for both rotators. Coarse rotation is imparted to the telescope through a flexible cable which is tensioned only during use. The fine motion uses a tangent arm actuated by a ball screw. The arm is preloaded by a small, free hanging weight which virtually eliminates backlash and/or compliance. The coarse rotator is operated open-loop, and usually positions the telescope to about 0.25 degree. The least significant bit of the fine motion corresponds to 1.5 arc seconds, and final settings are accurate, on the average, to 3 arc seconds when circle division errors are accounted for.

Once set, the telescope is clamped during observations by a DC Torquer compressing a split ring with a screw. Constant compression is assured by the constancy of the stall torque of the motor.

\section{The Divided Circle Scanning System}

The photodiode circle scanners are similar to types previously used on the six-inch transit circle except for the illumination of the circle 
and the method of defining the position of a division line of the circle. The illuminators use incandescent bulbs with integral lenses and beam splitters rather than the former flash tubes. The position of a line is defined by the output of an electronic filter. The filter is a weighting device wherein the weights are proportional to the derivative of the nominal profile of a division. The output of the filter passes through zero when the two halves of a division are in balance, thus defining the line center. Repeatability of a single scanner varys from 0.02 to 0.04 arc seconds. Six scanners, on three diameters, are used in the system.

The Solar Attachment

To observe the sun with the IDM operating in the same mode as for relatively fainter objects, and to reduce the insolation of the telescope, a neutral density filter 3 inches in diameter is mounted in front of the stopped-down objective for solar observations. The transmitted flux approximates that of the moon, and no additional attenuating filters are required. The filter's surfaces are flat to $1 / 20$ wave to reduce residual power, and the filter is rotated 180 degrees about the optical axis at the mid-point of a solar limb observation to eliminate any wedge effects. See Figure 4 .

\section{The Clock System}

The basic system consists of a quartz oscillator, a VLF receiver/ phase comparator, and a sidereal converter. This basic unit is tripled to achieve double redundancy. Various self-checks are used, e.g., the beat frequency between a mean time oscillator and its associated sidereal converter is monitored for constancy. Two cesium beam oscillators have recently been added to allow the recording of TAI for observations of solar system objects. The time associated with, for example, an IDM raster scan, is derived from a counter with a 20 micro-second resolution. It is triggered at a known clock time and reset appropriately. Digital time is available to the controller from all the clocks. The entire clock system is monitored through a coaxial switching array either manually, or by the HP controller next described.

\section{Control and Data Acquisition}

An HP 21MX-F controller with disc, tape, printer, terminals and other peripherals controls the observing system and records data from the telescope, the clock system and other sources such as environmental sensors. All operations connected with observing are routed through the controller, although some overrides are available if required, e.g., roof motor control.

A queue of objects to be observed is entered, each object identified by a five digit number, and automatic operation then ensues. As each object is to be observed a refracted apparent place is calculated, the telescope appropriately set, and IDM and circle scanner operations initiated. Using the predicted position it is possible to acquire an object within 
a 5" square area. A wealth of status information concerning the entire operation is available to the observer. Most errors are detected and reported by the system in real time. The observer communicates with, and monitors, the system by means of a CRT terminal and three TV monitors displaying, respectively; a closed circuit picture of the remote telescope, a section of the divided circle, and IDM digital data converted to an 8-level gray scale. The latter display is heavily used for diagnostic purposes, and in such cases aids, such as joystick control of the position of the scanned raster, are available.

The operating system supports multi-tasking, and in addition to the tasks just described, it is used to produce in situ observational results, to carry out analyses of instrumental behavior, and for other scientific projects of the assigned personnel.

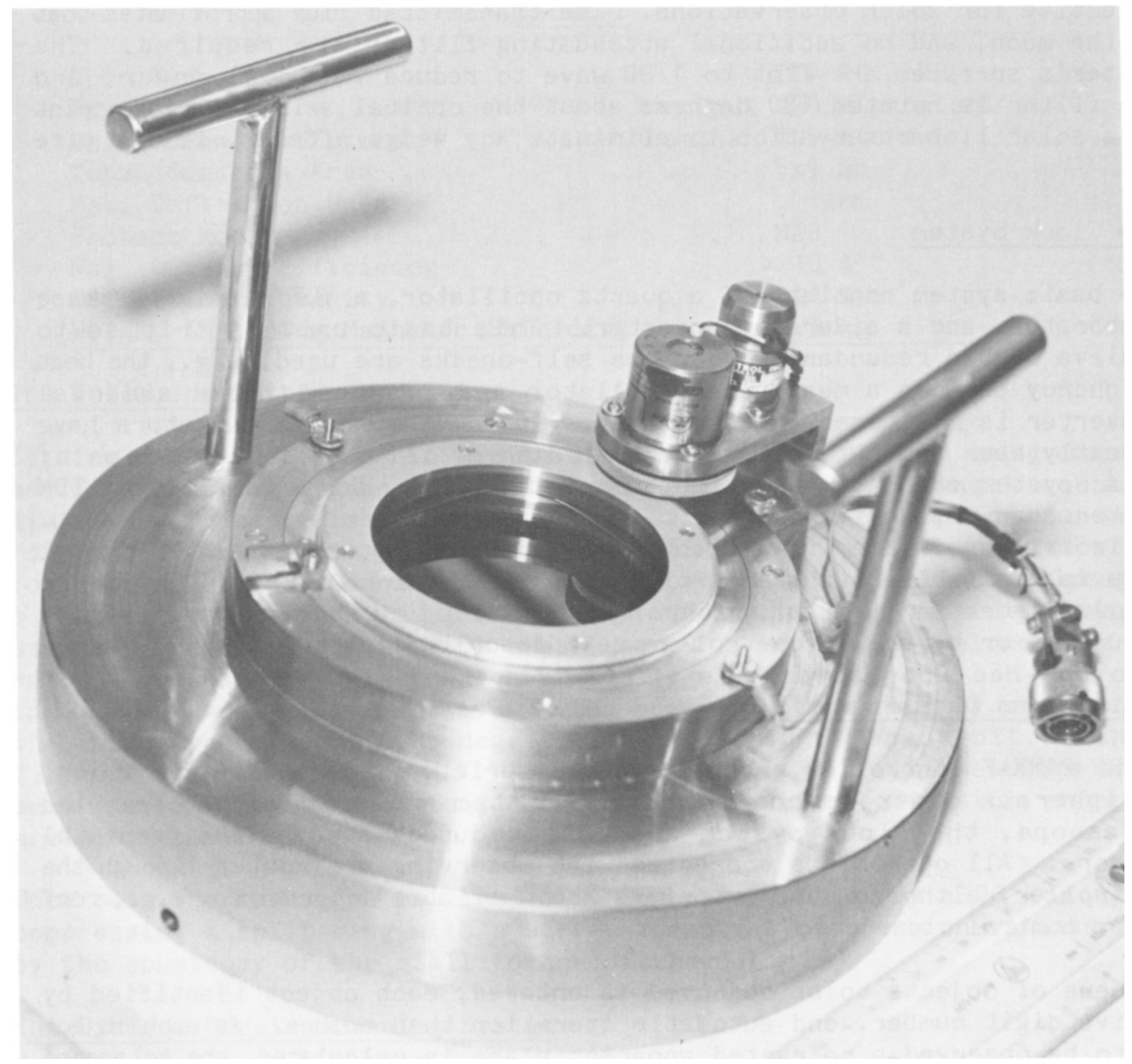

Figure 4 - Solar Attachment 
Image Centroiding Algorithms

The digital stellar and wire images produced by the IDM are processed in real time with centroiding errors of $+/-0.03$ arc second or less. A single raster scan (or "snapshot") is processed in 150 milliseconds. After experimentation with functional fits, i.e., a two dimensional Gaussian for point sources and the Sinc function for the wires, a simpler moment analysis was finally adopted. It is fast, converges well, and has been found to be more accurate than the full function fit for low S/N ratios such as the IDM produces for faint objects. A background level is determined and removed. This is particularly important for day stars. Night and day star data are shown in Figures 5 and 6 respectively. Note that the computer printer compresses these images in the "y" direction.

The images of Uranus, Neptune and Mercury are processed by the stellar algorithm, with the latter planet later corrected for phase effects. Mars, Jupiter and Saturn are processed by fitting a functional curve to the distribution of edge pixels. These pixels are defined by a threshold set approximately at 2.5 sigma above the local background. This is equivalent to the local Laplacian indicating a significant curvature of the background. Sampling scans are made from the four cardinal directions, and the median of the threshold levels is used to contour the image. The pixels are further identified as being either on the sunward or phaseward side of the image. For Mars and Jupiter an iterative least squares ellipsoidal fit is made involving five unknowns and two or three iterations. The standard deviation of consecutive test scans of Jupiter, for example, has been about $+/-0.4$ arc seconds, and forty to over one hundred such scans are possible within a single observation. Saturn is modeled by fitting a general second order function of two variables to the outer portion of the rings, unless the latter are edge on, in which case the planetary disc itself is used. See Figures 7 and 8.

The most difficult planet is Venus, due to its large phase effects and to the day sky background. In this case a circle of assumed radius has been fit to the sunward edge pixels. The value of the radius being taken from the ephemeris. Overall, the center can be determined to about $+/-$ 1 arc second in a single snapshot, and as noted above, forty to one hundred or more such scans are possible during a transit.

The stellar centroiding routine provides a great deal of information in addition to the $\mathrm{x}$ and $\mathrm{y}$ positions. Such information includes, for example, the Full Width at Half Maximum (FWHM) for a stellar image, which is a good measure of the astronomical seeing. A 16-bit status word is also available which gives diagnostic information about the observation. 
IMACE DISSECTOR SCAN OF POLARIS SHOWING THE RAW COUNTS

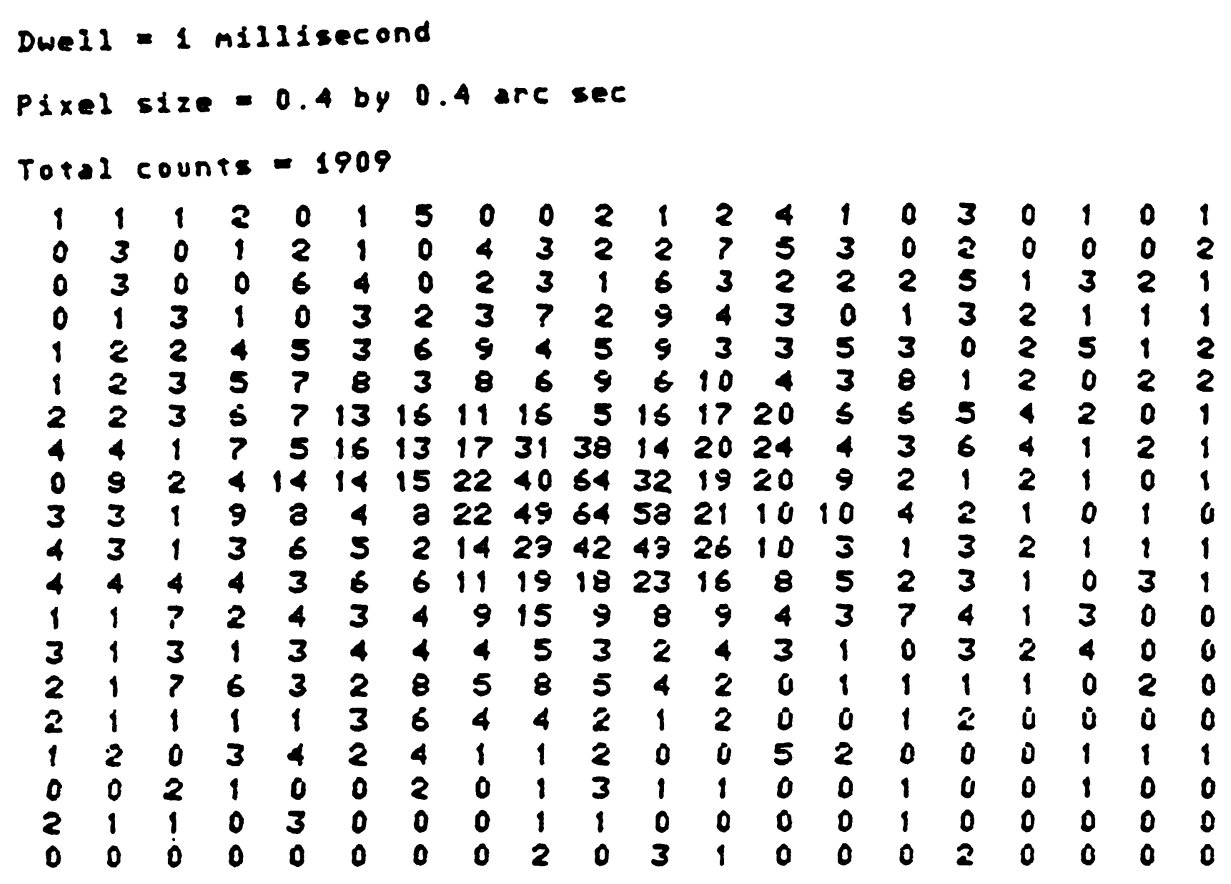
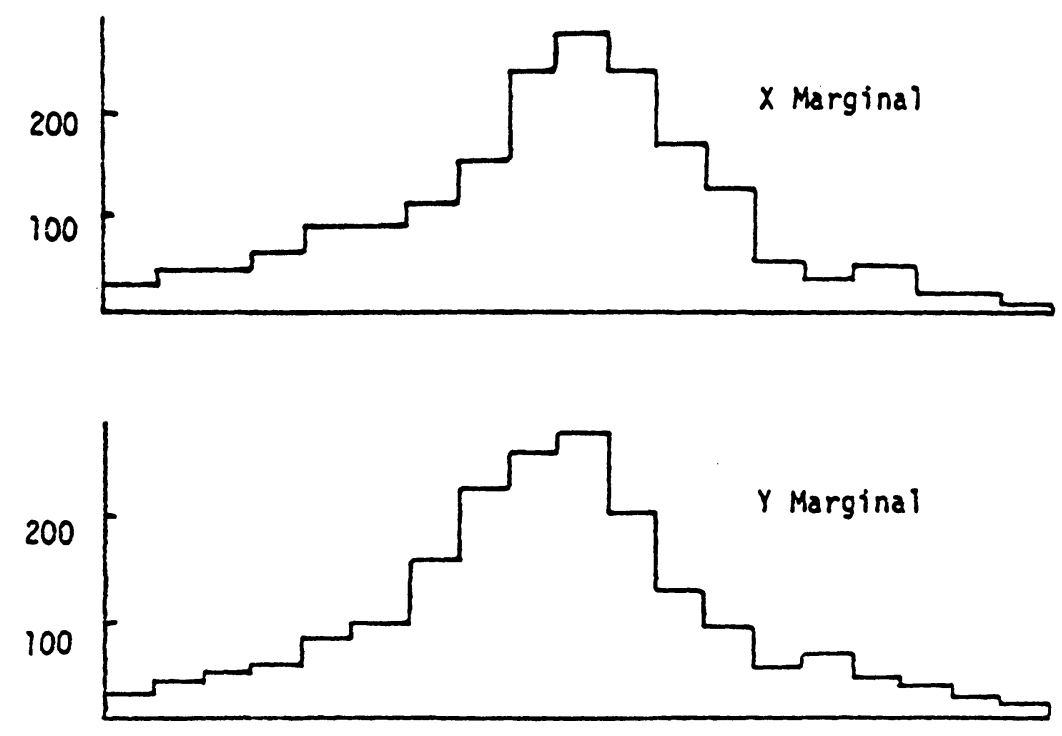

Figure 5 
IMAGE DISSECTOR SCAN OF THE DAYSTAR POLARIS

\section{WITH BACKGROUND SUPPRESSION}

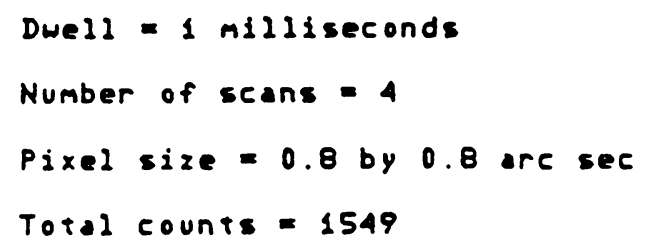

$\begin{array}{lllllllllllllllllllll}0 & 0 & 0 & 0 & 0 & 0 & 0 & 0 & 0 & 0 & 0 & 0 & 0 & 0 & 0 & 0 & 0 & 0 & 0 & 0 \\ 0 & 0 & 0 & 0 & 0 & 0 & 0 & 0 & 0 & 0 & 0 & 0 & 0 & 0 & 0 & 0 & 0 & 0 & 0 & 0 \\ 0 & 0 & 0 & 0 & 0 & 0 & 0 & 0 & 0 & 0 & 0 & 0 & 0 & 0 & 0 & 0 & 0 & 0 & 0 & 0 \\ 0 & 0 & 0 & 0 & 0 & 0 & 0 & 0 & 0 & 0 & 0 & 0 & 0 & 0 & 0 & 0 & 0 & 0 & 0 & 0 \\ 0 & 0 & 0 & 0 & 0 & 0 & 0 & 0 & 0 & 0 & 0 & 0 & 0 & 0 & 0 & 0 & 0 & 0 & 0 & 0 \\ 0 & 0 & 0 & 0 & 0 & 0 & 0 & 0 & 0 & 0 & 0 & 0 & 0 & 0 & 0 & 0 & 0 & 0 & 0 & 0 \\ 0 & 0 & 0 & 0 & 0 & 0 & 0 & 0 & 0 & 0 & 52 & 0 & 0 & 0 & 0 & 0 & 0 & 0 & 0 & 0 \\ 0 & 0 & 0 & 0 & 0 & 0 & 0 & 0 & 46 & 62 & 81 & 41 & 0 & 0 & 0 & 0 & 0 & 0 & 0 & 0 \\ 0 & 0 & 0 & 0 & 0 & 0 & 39 & 67 & 69 & 68 & 82 & 63 & 38 & 40 & 0 & 0 & 0 & 0 & 0 & 0 \\ 0 & 0 & 0 & 0 & 0 & 0 & 39 & 53 & 64 & 67 & 65 & 62 & 64 & 53 & 0 & 0 & 0 & 0 & 0 & 0 \\ 0 & 0 & 0 & 0 & 0 & 0 & 0 & 0 & 0 & 46 & 41 & 54 & 67 & 0 & 0 & 0 & 0 & 0 & 0 & 0 \\ 0 & 0 & 0 & 0 & 0 & 0 & 0 & 0 & 0 & 0 & 0 & 45 & 41 & 0 & 0 & 0 & 0 & 0 & 0 & 0 \\ 0 & 0 & 0 & 0 & 0 & 0 & 0 & 0 & 0 & 0 & 40 & 0 & 0 & 0 & 0 & 0 & 0 & 0 & 0 & 0 \\ 0 & 0 & 0 & 0 & 0 & 0 & 0 & 0 & 0 & 0 & 0 & 0 & 0 & 0 & 0 & 0 & 0 & 0 & 0 & 0 \\ 0 & 0 & 0 & 0 & 0 & 0 & 0 & 0 & 0 & 0 & 0 & 0 & 0 & 0 & 0 & 0 & 0 & 0 & 0 & 0 \\ 0 & 0 & 0 & 0 & 0 & 0 & 0 & 0 & 0 & 0 & 0 & 0 & 0 & 0 & 0 & 0 & 0 & 0 & 0 & 0 \\ 0 & 0 & 0 & 0 & 0 & 0 & 0 & 0 & 0 & 0 & 0 & 0 & 0 & 0 & 0 & 0 & 0 & 0 & 0 & 0 \\ 0 & 0 & 0 & 0 & 0 & 0 & 0 & 0 & 0 & 0 & 0 & 0 & 0 & 0 & 0 & 0 & 0 & 0 & 0 & 0 \\ 0 & 0 & 0 & 0 & 0 & 0 & 0 & 0 & 0 & 0 & 0 & 0 & 0 & 0 & 0 & 0 & 0 & 0 & 0 & 0 \\ 0 & 0 & 0 & 0 & 0 & 0 & 0 & 0 & 0 & 0 & 0 & 0 & 0 & 0 & 0 & 0 & 0 & 0 & 0 & 0\end{array}$

Figure 6 
Dwell = 1 milliseconds

Pixel size $=1.2$ by 1.2 are sec Total counts $=19580$

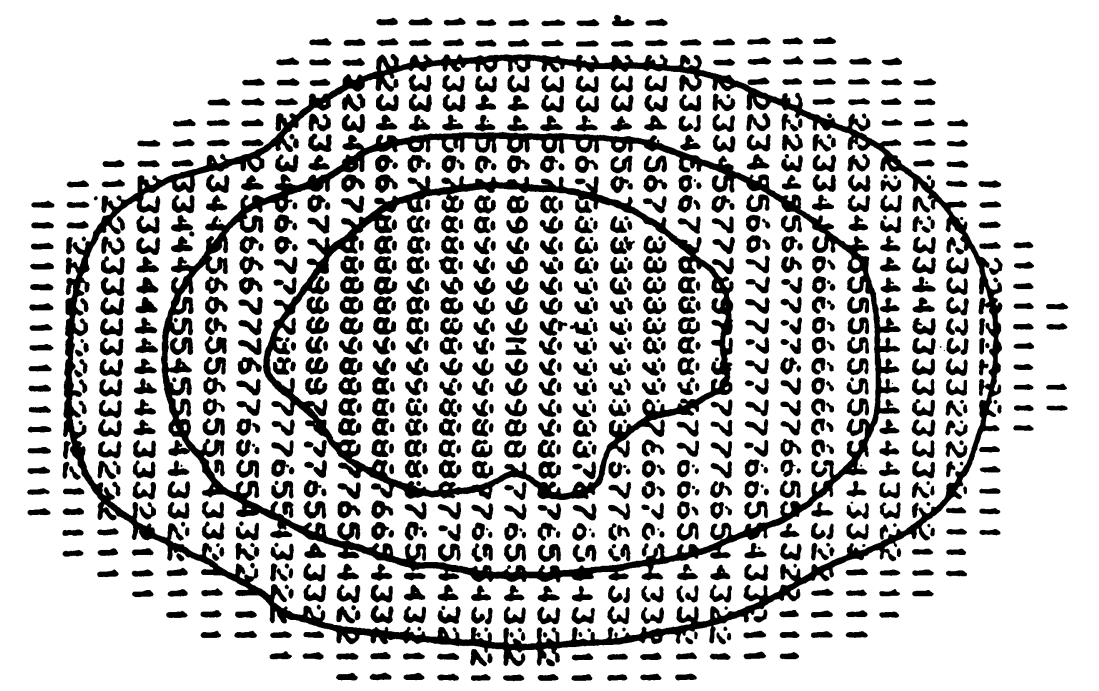

EDGE DETECTION PATTERN FOR JUPITER AT A CONTOUR

LEVEL OF 25 PER CENT OF PEAK INTENSITY

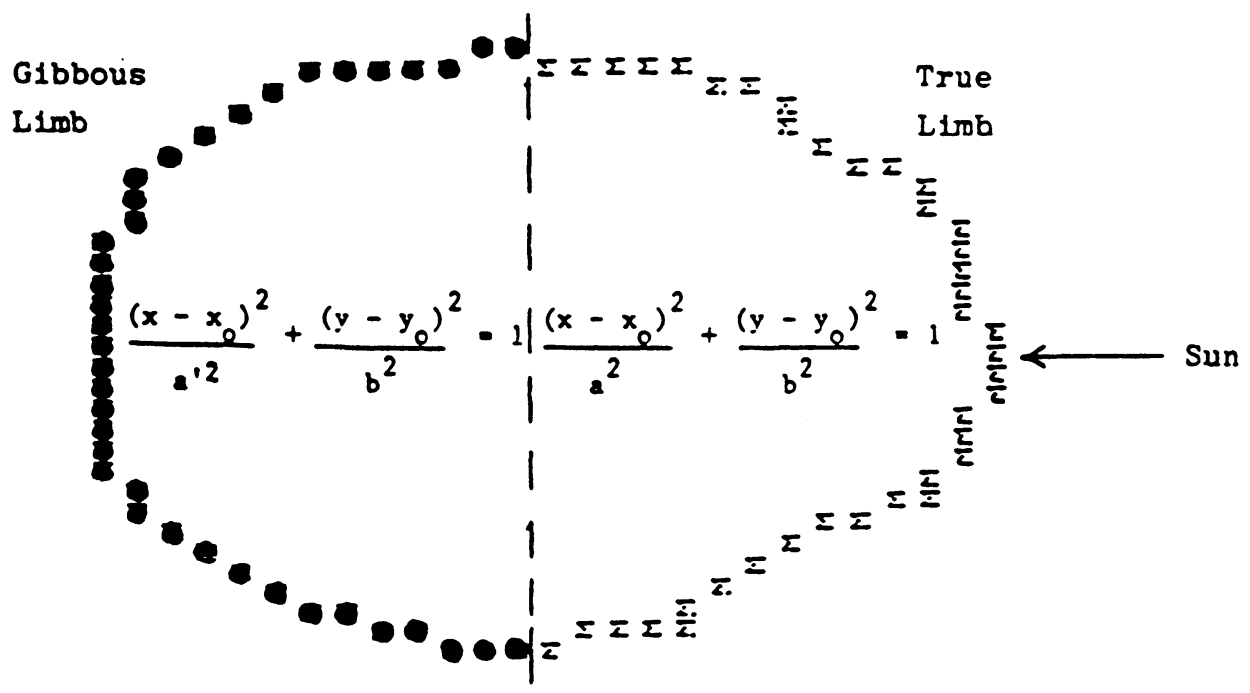

Figure 7 
DIGITAL IMAGE FOR THE PLANET SATURN

Dwell = 1 milliseconds

Pixel size $=1.2$ by 1.2 are sec Total counts $=9147$

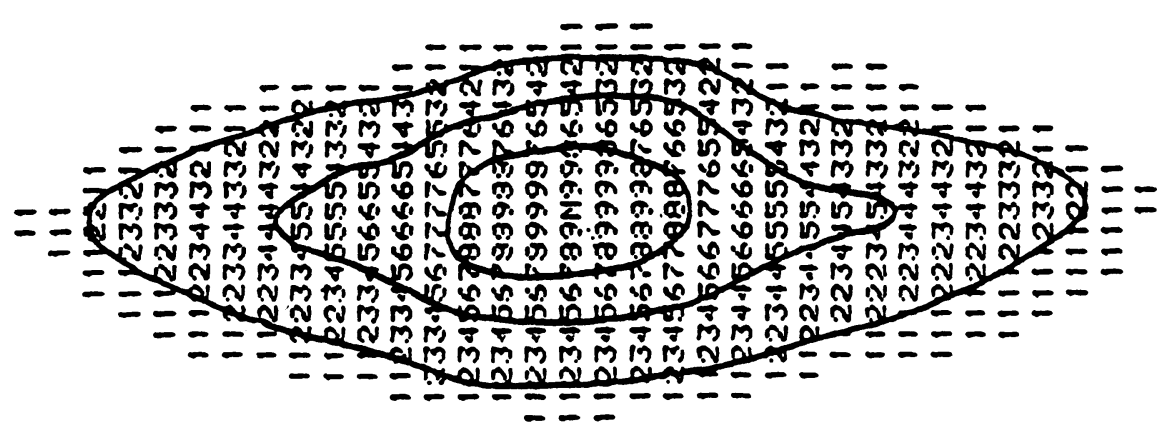

EDGE DETECTION PATTERN FOR SATURN AT A CONTOUR

LEVEL OF 25 PER CENT OF PEAK INTENSITY

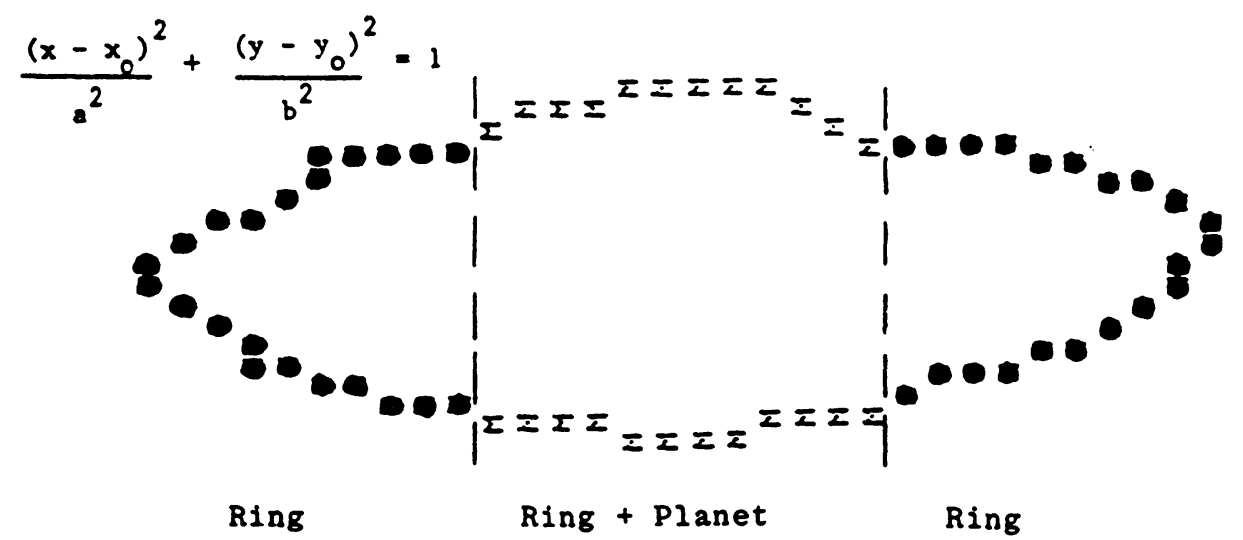

Figure 8 


\section{THE NEW ZEALAND PROGRAM}

\section{Rationale}

At the conclusion of the observing phase of the international Southern Reference Stars (SRS) program, suspected deficiencies of the fundamental system in the southern sky were fully confirmed. Indeed, it became evident that the magnitudes assigned to previously recognized errors were unduly optimistic. Also, considering the observational histories of the SRS, it has long been acknowledged that second epoch observations will be absolutely necessary if acceptable proper motions are to be derived.

Because of the two preceding facts, a large scale absolute observing program in the southern hemisphere, coupled with differential reobservations of the SRS, is an astrometric necessity. For this reason the U.S. Naval Observatory is sending its seven-inch transit circle to the Black Birch Astrometric Observatory, near Blenheim, New Zealand, at latitude 42 degrees $S$ and elevation 1500 meters. Observing there is scheduled for ten years, and will be conducted in tandem with a similar program of the six-inch transit circle in Washington. A simultaneous, coordinated program of pole-to-pole absolute observations will take place for the first time in astrometric history. The six-inch will reobserve the AGK3R stars. (It may be recalled that the SRS and AGK3R lists are referred to collectively as the International Reference Stars (IRS).) Thus the total observing list will include some 5,000 FK5 and 40,000 IRS plus major and minor planets, and the sun and the moon.

\section{Status}

The diplomatic understandings necessary for the conduct of the New Zealand program have been concluded, and the construction of the facility is essentially complete. The first shipments of material from the Naval Observatory have already arrived in New Zealand, and the first personnel will arrive, coincidentally, at the close of this meeting. The transit circle proper and the balance of the personnel will follow in February and March. New Zealand citizens will take part in the program to the maximum extent possible. A twin astrograph will also be installed (1986), but its program is not considered here. The seven-inch transit circle telescope and the Black Birch site in preparation are shown in Figures 9 and 10 .

\section{Purpose}

It may be appropriately mentioned here that these programs are important parts of larger strategy culminating in a fundamental compiled catalog in the mid-1990's. This catalog will be based upon an ab initio compilation of selected data with the subject programs furnishing data for the latest epochs. The IRS will also be available in the resulting system.

Besides the usual applications of such a catalog, the astrometric community 


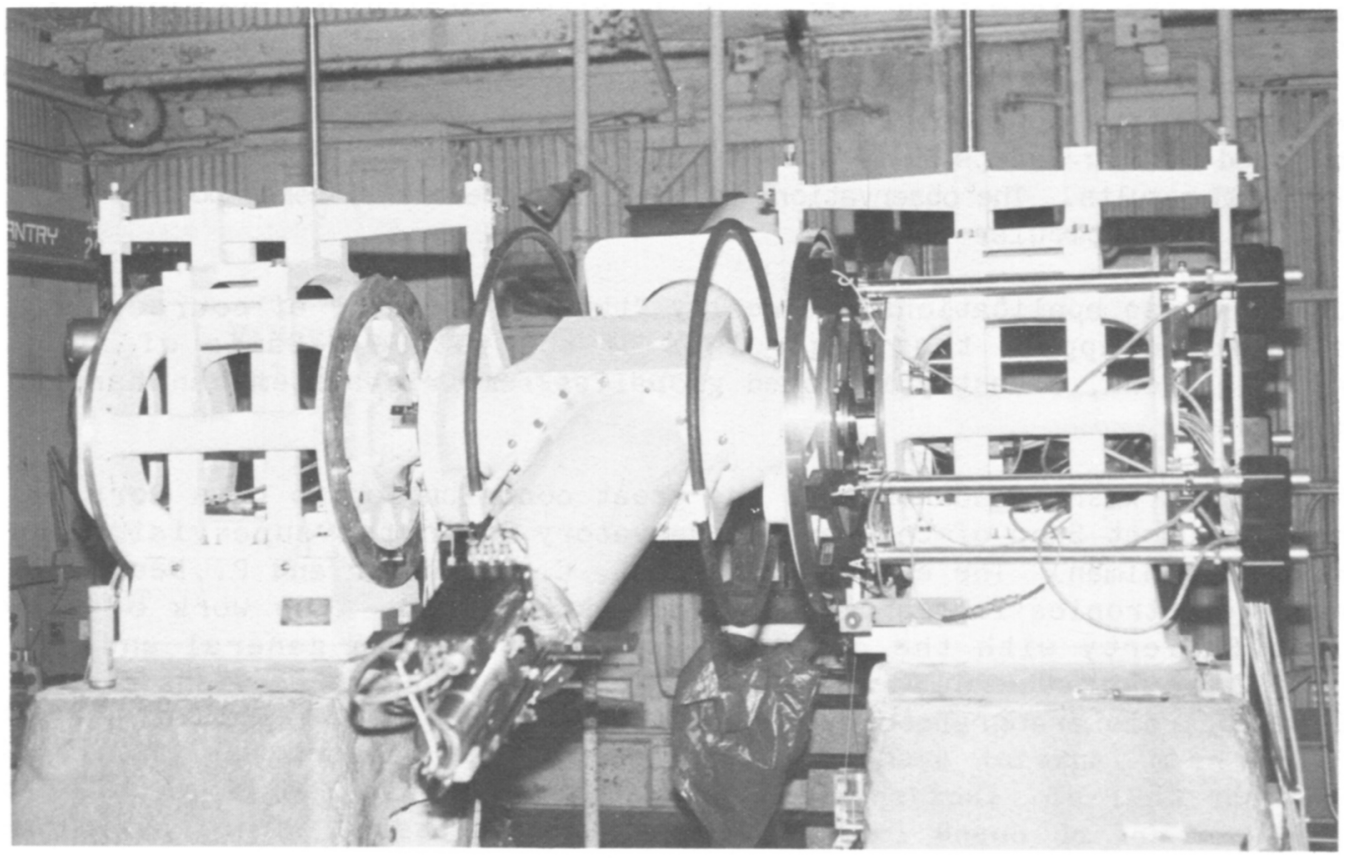

Figure 9 - The Seven-inch Transit Circle

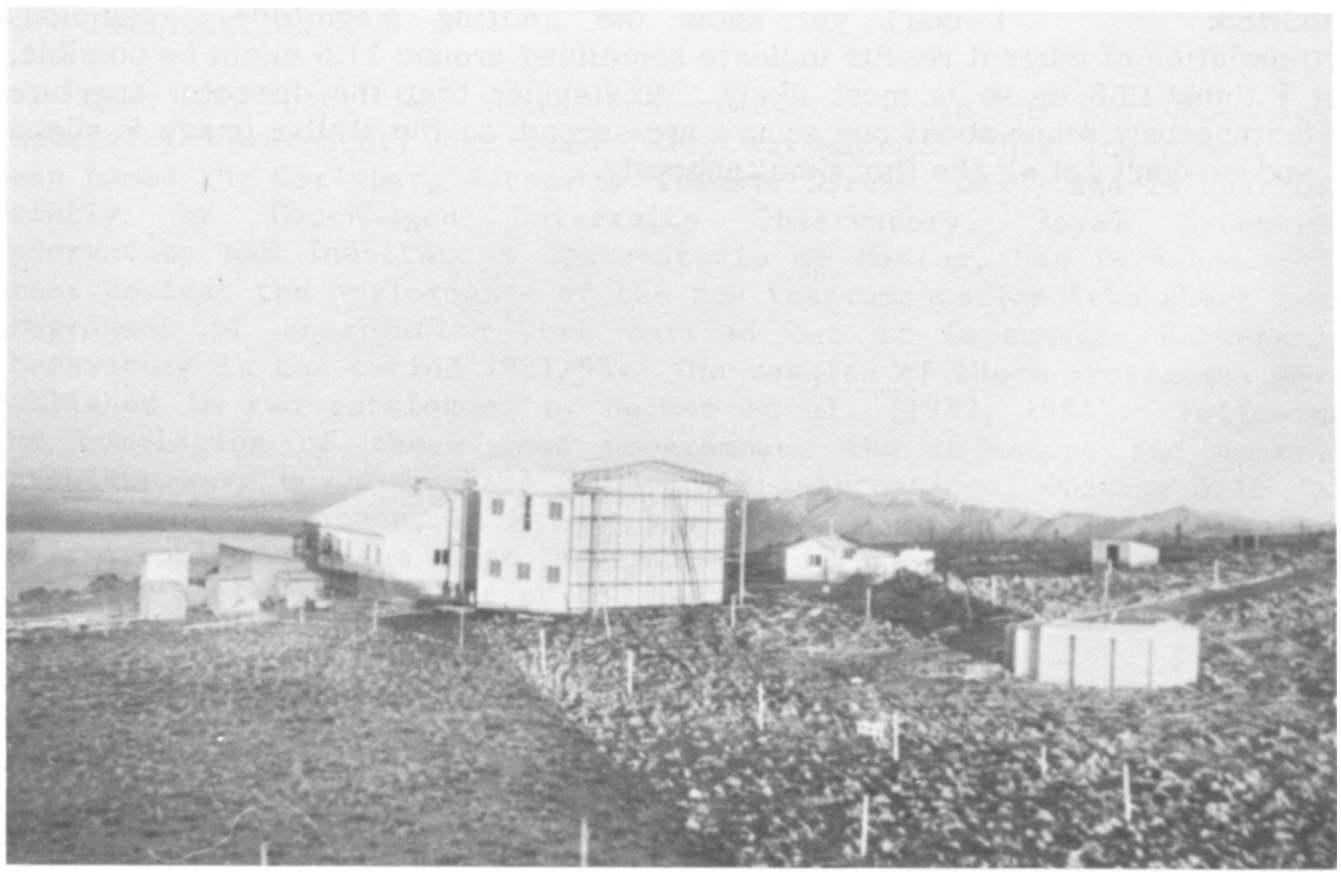

Figure 10 - The Black Birch Site 
will find the information most helpful in two particular instances: 1) The discovery and elaboration of any systematic tendencies of the techniques of which we are hearing at this meeting, particularly the precise interferometric approaches at the various wavelengths, and 2) Satisfying the need for an independent standard for comparision with the anticipated HIPPARCOS results. The observations of the IRS, essentially contemporaneously, will be of particular value.

Both of these applications are really "two way streets" of course, and so it would appear that regardless of the future details of such investigations, a vastly improved global astrometric system can hardly be avoided!

The authors wish to acknowledge the great contribution to this work by the Instrument Shop of the Naval Observatory under the supervision of Mr. John Pohlman. The efforts of Messrs. C. Carpenter and P. Heermans in the electronics laboratory are also acknowledged. The work of $\mathrm{Mr}$. T. J. Rafferty with the circle scanning system in general and his supervision of the determination of the diameter corrections of the divided circle are gratefully acknowledged.

\section{Discussion:}

HDG:

HUGHES: I don't yet know the limiting magnitude. Judicious extrapolation of current results indicate something around $11.5 \mathrm{might}$ be possible, but I think 10.5 or so is most likely. Remember that the dissector aperture instantaneously scans about one square arc-second, so the stellar image is sliced up and we don't get all the flux simultaneously. 